\title{
Evaluation of Self-Collimated Beams in Photonic Crystals for Optical Interconnect
}

\author{
Tsuyoshi Yamashita, Member, IEEE, and Christopher J. Summers, Senior Member, IEEE
}

\begin{abstract}
Self-collimated beams and photonic bandgap mirrors in photonic crystals are evaluated for applicability in an on-chip interconnect system. Simulations using the plane-wave expansion and finite-difference time-domain methods are utilized to design and evaluate the theoretical performance of these systems, called a virtual waveguide due to borderless confinement of the signal. The effect of systematic and random fabrication errors on the performance is characterized. Coupling efficiency is virtually unaffected by misalignment, but is found to be a strong function of the length of the waveguide and the frequency of light. Additional routing capabilities of sharp $90^{\circ}$ turns and signal crossings with no crosstalk are demonstrated. Photonic crystal virtual waveguides are ideal structures for on-chip optical signal routing.
\end{abstract}

Index Terms-Integrated optics, optical interconnect, photonic bandgap, photonic crystals, self-collimation.

\section{INTRODUCTION}

$\mathbf{T}$ WO OF THE major challenges of integrating optical devices onto a chip are the difficulty of routing an optical signal and the high sensitivity to misalignment when coupling into a waveguide. In addition, crosstalk between waveguides in proximity, losses from sharp turns, and cross-coupling when signals intersect make optical routing difficult. The unique dispersion property of a square lattice photonic crystal slab is investigated to address these issues.

Photonic crystals display photonic band properties similar to semiconductors such as bandgaps and defect modes [1]-[3]. Analogous to the Fermi surfaces for electronic band structure, the allowed wavevector curve in a photonic crystal reveals the $\mathbf{k}$ vectors and, thus, the propagation direction, of photons with a specified energy. When the allowed wavevector curve becomes linear, self-collimation occurs where an optical beam is restricted from spreading [4]-[8]. A virtual waveguide, where an optical beam is confined by the photonic crystal structure without any additional physical boundaries is formed. In addition, it is possible to make sharp $90^{\circ}$ bends utilizing interfaces with total internal reflection [7], [8].

This paper outlines the design process of photonic crystal virtual waveguides using simulation data of structures made from silicon and silica for application in on-chip interconnects. Additional simulations are conducted to evaluate the characteristics of the virtual waveguide system.

Manuscript received June 30, 2004; revised Septembr 221, 2004. This work was supported in part by the National Science Foundation's Graduate Research Fellowship and in part by the the MultiUniversity Research Initiative sponsored by the Army Research Office under Contract DAAD19-01-1-0603.

The authors are with the School of Material Science and Engineering, Georgia Institute of Technology, Atlanta, GA 30332-0245 USA (e-mail: gte532f@mail.gatech.edu; chris.summers@mse.gatech.edu).

Digital Object Identifier 10.1109/JSAC.2005.851200

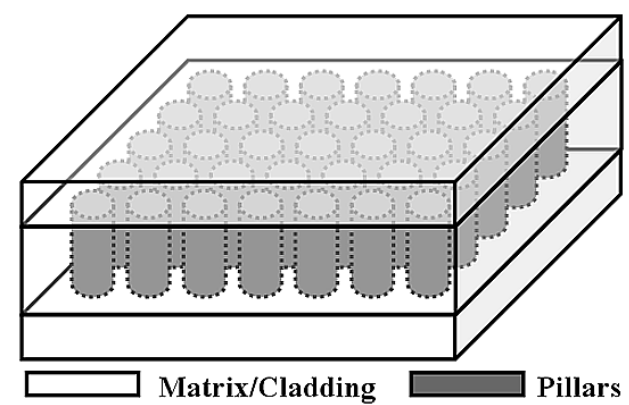

Fig. 1. Geometry of the photonic crystal structure investigated for the virtual waveguide.

\section{THEORY AND DESIGN}

Photonic crystals are a class of periodic media, where the dielectric constant is modulated in one, two, or three dimensions. Fig. 1 shows an example of a two-dimensional (2-D) photonic crystal slab structure that is investigated in this paper. This structure consists of cylindrical pillars arranged in a square lattice. Confinement in the third dimension can be achieved through index contrast as in an ordinary slab waveguide. An effective media method allows 2-D simulations to provide useful conclusions for this three-dimensional (3-D) structure [9]-[11]. The effective media method results in lower indices for the materials corresponding to mode structure in the slab. Structures using silicon $(\varepsilon=11)$ and silica $(\varepsilon=2)$ are chosen to ensure compatibility with conventional semiconductor processes where the dielectric constant of silicon is taken lower than its value of 12.1 at $1.55 \mu \mathrm{m}$ to account for the out of plane confinement. The propagation of electromagnetic waves in a photonic crystal is characterized using Maxwell's equations in linear media with no sources

$$
\begin{aligned}
\nabla \times \vec{E} & =-\mu \frac{\partial \vec{H}}{\partial t} \\
\nabla \times \vec{H} & =\varepsilon \frac{\partial \vec{E}}{\partial t} \\
\nabla \cdot \vec{E} & =\nabla \cdot \vec{H}=0
\end{aligned}
$$

where $E$ and $H$ are the electric and magnetic fields and $\varepsilon$ and $\mu$ are the dielectric constant and magnetic permeability. In this paper, two numerical methods were used to solve the Maxwell's equations in the photonic crystal: the plane-wave expansion (PWE) method and the finite-difference time-domain (FDTD) method. 


\section{A. PWE Method}

In the PWE method, the band diagram of a photonic crystal, which plots the wavevector $(\mathbf{k})$ versus the frequency $(\omega)$ of electromagnetic waves that propagate in the photonic crystal, is solved. The Maxwell's equations are rearranged to depend only on the $E$ or the $H$ field in the form:

$$
\frac{1}{\varepsilon(\vec{r})} \nabla \times\{\nabla \times \vec{E}(\vec{r})\}=\frac{\omega^{2}}{c^{2}} \vec{E}(\vec{r})
$$

where $c$ is the speed of light. The dielectric constant and the electric field are now periodic functions in space. Maxwell's equations are solved by expanding each periodic function in reciprocal (k) space and solving a system of equations numerically to calculate the relationship between $\omega$ and $\mathbf{k}$. In this paper, 625 terms of the expansion are used to provide sufficient accuracy for design purposes. The band diagram and the allowed wavevector curves are plotted inside the first Brillouin zone in the 2-D square lattice. Once the $\omega$ versus $\mathbf{k}$ relationship is solved, the direction of energy propagation is found by taking the normal direction to the allowed wavevector curve, or the constant $\omega$ curve in $\mathrm{k}$ space. The curvature of the allowed wavevector curve determines the degree of beam spread inside of the photonic crystal. Therefore, a linear section in the curve gives rise to self-collimated beams traveling in a direction normal to the line. In addition, frequencies where there are no $\mathbf{k}$ values in the solution of the Maxwell's equation correspond to bandgaps, where the photonic crystal behaves as a perfect mirror. Since Maxwell's equations scale with the length scale of the unit cell, a normalized frequency is used, $\omega_{n}=\omega a / 2 \pi c$, where $a$ is the lattice constant of the photonic crystal. Normalization allows the results to be interpreted and scaled to fit a design wavelength.

The geometry in Fig. 1 is simulated using silicon pillars in silica and silica pillars in silicon. The band structures and allowed wavevector curves for the pillar radii varying from 0 to $0.5 a$ are calculated. The structure with silicon pillars of radius $0.2 a$ in a silica matrix is found to exhibit strong self-collimation in the $\boldsymbol{\Gamma}-\mathbf{M}$ direction on the top edge of the first band for the TE polarization. The band diagram and allowed wavevector curves for this structure are shown in Figs. 2 and 3.

As shown in Fig. 2, between normalized frequencies of 0.28 and 0.33 , a bandgap exists where any incident beam will be totally reflected. The section of the band diagram below the bandgap and between the $\mathbf{X}$ and $\mathbf{M}$ frequencies $\left(\omega_{n}=0.24-0.28\right)$ exhibits self-collimation. The allowed wavevector curves, shown in Fig. 3, change from convex to concave along the $\boldsymbol{\Gamma}-\mathbf{M}$ line (dashed bold line) as the frequency is increased from $\omega_{n}=0.24$ to $\omega_{n}=0.28$. The allowed wavevector curve for $\omega_{n}=0.26$ is completely flat and normal to the $\boldsymbol{\Gamma}-\mathbf{M}$ line. Therefore, a Gaussian beam incident on the photonic crystal along the diagonal of the unit cell with a normalized frequency of 0.26 is expected to exhibit the highest degree of self-collimation.

The most common wavelengths for optical signals are $850 \mathrm{~nm}, 1.3 \mu \mathrm{m}$, and $1.55 \mu \mathrm{m}$. However, since silicon absorbs light below $1.06 \mu \mathrm{m}$, only the $1.3 \mu \mathrm{m}$ and $1.55 \mu \mathrm{m}$ cases can be considered. The lattice constant $a$, to achieve self-collimation for the design wavelength is determined by equating the design wavelength to a normalized frequency of 0.26 . The formula for

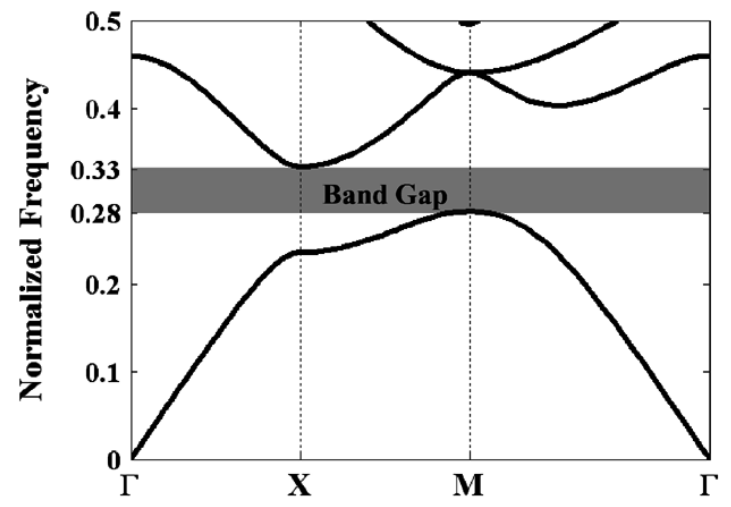

Fig. 2. TE mode band diagram of the square lattice photonic crystal of silicon pillars in a silica matrix with radii of $0.2 \mathbf{a}$ in the reduced Brillouin zone.

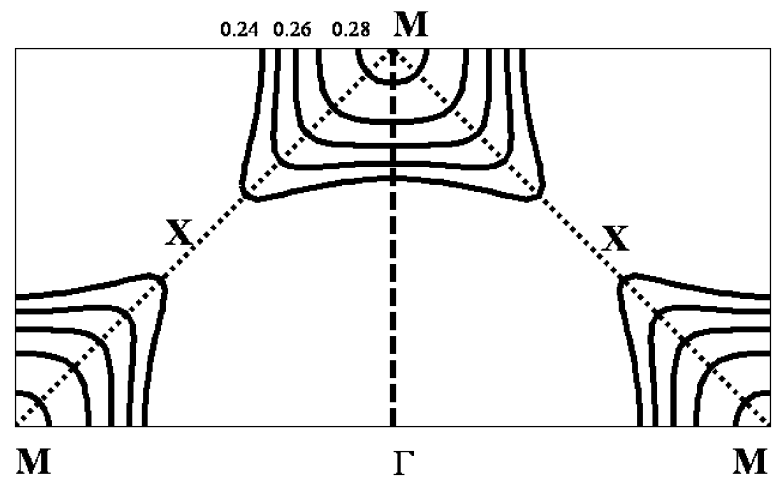

Fig. 3. Allowed wavevector curve showing a flattening of the curve along the $\boldsymbol{\Gamma}$-M direction resulting in a self-collimated beam at a normalized frequency of 0.26 .

the normalized frequency is rearranged to find that $a=\omega_{n} \lambda$, where $\lambda$ is the wavelength of the incident radiation. The lattice constants are $0.338 \mu \mathrm{m}$ and $0.403 \mu \mathrm{m}$ for $1.3 \mu \mathrm{m}$ and $1.55 \mu \mathrm{m}$ wavelengths, respectively. The silicon pillars $(r=0.2 a)$ have diameters of $135.2 \mathrm{~nm}$ and $161.2 \mathrm{~nm}$, respectively, which are within the processing capability of state of the art lithography. The values for $1.55 \mu \mathrm{m}$ incident wavelength will be used for the rest of this paper, but all conclusions can be directly applied to a system with $1.3 \mu \mathrm{m}$ wavelength by scaling the structure accordingly.

\section{B. Finite-Difference Time-Domain (FDTD) Method}

The second simulation method used in this paper is the FDTD method. In this method, Maxwell's equations are discretized so that the $E$ and $H$ fields are solved from the $E$ and $H$ fields at a previous time step and the material constants of a grid. The 2-D TE mode FDTD is utilized in this paper

$$
\begin{aligned}
\frac{\partial H_{x}}{\partial t} & =\frac{1}{\mu}\left[-\frac{\partial E_{z}}{\partial y}\right] \\
\frac{\partial H_{y}}{\partial t} & =\frac{1}{\mu}\left[\frac{\partial E_{z}}{\partial x}\right] \\
\frac{\partial E_{z}}{\partial t} & =\frac{1}{\varepsilon}\left[\frac{\partial H_{y}}{\partial x}-\frac{\partial H_{x}}{\partial y}\right] .
\end{aligned}
$$

Each field component is updated from the data at the previous time step. The performance of the virtual waveguide is simulated by following the $E$ and $H$ field values as a Gaussian beam, 

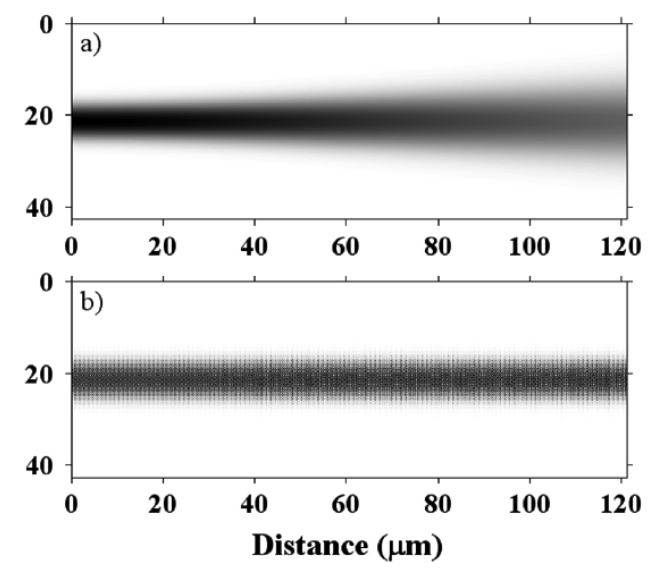

Fig. 4. Gaussian beam with normalized frequency of 0.26 propagating in (a) silica and (b) photonic crystal virtual waveguide. Significant spreading is seen in silica while the photonic crystal keeps the beam intact.

initiated inside of the grid, travels through, reflects from, refracts in, and resonates inside of the photonic crystal. The grid is bound by a Berenger's perfectly matched layer (PML) to absorb any radiation that propagates to the edge of the simulation grid. Lossless media are used in the simulation.

The Gaussian beam full width at half max (FWHM) used throughout this paper is 300 grid points wide or about $8.5 \mu \mathrm{m}$ for the $1.55 \mu \mathrm{m}$ incident wavelength. This width corresponds to mode field radii of some single mode fibers available and is a realistic beamwidth for analysis. To verify the self-collimation predicted by the PWE simulation, an FDTD simulation grid is created using 20 grid points for each side of the nonprimitive unit cell containing two pillars diagonally placed for propagation in the $\Gamma-\mathbf{M}$ direction. The Courant stability limit is utilized for the time step. Fig. 4 depicts the plot of the time-averaged electric field intensity pattern of a Gaussian beam propagating in silica compared with inside of a virtual waveguide for normalized frequency of 0.26 . After propagation through $120 \mu \mathrm{m}$, or 4250 grid points, no observable spreading occurs in the virtual waveguide compared with significant spreading in the silica slab.

A combination of PWE and FDTD methods is used to evaluate the performance of the photonic crystal virtual waveguide consisting of the silicon pillars in a silica matrix.

\section{RESULTS AND DISCUSSION}

\section{A. Tolerance to Fabrication Errors}

Many proposed integrated optics devices rely on resonance for achieving their functionality. This factor contributes to very stringent fabrication tolerances that make applications of these devices difficult beyond the simulation realm. Photonic crystals also suffer from this difficulty in applications utilizing defect resonant modes, where a small deviation in size or spacing can dramatically change the performance of the device. However, self-collimation occurs in a photonic crystal over a range of frequencies, so small errors can be accommodated.

A FDTD grid measuring $3000 \times 7000$ grid points is filled with the photonic crystal except for a 70 grid wide region of isotropic silica denoting the input plane. The beamwidth

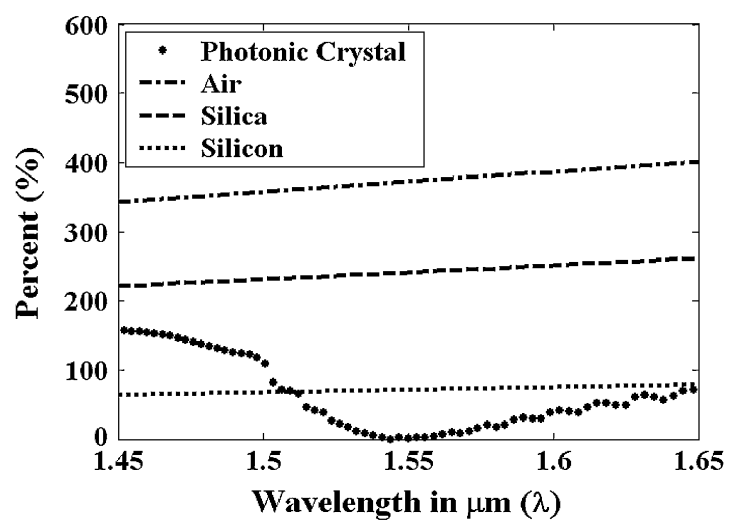

Fig. 5. Percentage of beam expansion after $171 \mu \mathrm{m}$ of propagation in a self-collimating photonic crystal compared with three isotropic media.

was recorded after propagation through 6000 grid points, or $171 \mu \mathrm{m}$, from the incident plane and compared with the incident beamwidth for a photonic crystal filled grid and with several isotropic media. The beamwidth expansion after traveling $171 \mu \mathrm{m}$ as a function of wavelength is plotted in Fig. 5 . The range of wavelengths where the beam spread is under $20 \%$ spans almost $50 \mathrm{~nm}$ centered on $1.55 \mu \mathrm{m}$. Self-collimated beams exhibit a dramatic decrease in the beam expansion over a wide frequency range compared with the case of pure air, silica, and silicon. The beam spreads in the isotropic media are calculated using the Gaussian beam spread equation

$$
w^{2}(z)=w_{0}^{2}\left[1+\left(\frac{\lambda_{0} z}{\pi n w_{0}^{2}}\right)^{2}\right]
$$

where $w_{0}$ is half of the FWHM at the beam waist, $w(z)$ is half of the FWHM after traveling a distance $z$, and $n$ is the index of refraction of the isotropic media.

Two types of errors are investigated in this study: systematic error and random error. Systematic error is modeled as a uniform increase/decrease of the pillar size which can result in fabrication from over or under development or exposure of the photoresist, as well as subsequent etch or deposition processes. All other factors remained constant compared with the previous simulation. Systematic errors of $\pm 5 \%$ and $\pm 2.5 \%$ are simulated and the results plotted in Fig. 6. For $2.5 \%$ error in either direction, the additional beam spread at $1.55 \mu \mathrm{m}$ is only $10 \%$. The general shape of the beam expansion curve remains constant, while larger pillars shift the minimum beam expansion toward larger wavelengths due to an increase in the average refractive index that lowers the band structure. The data of the $\pm 5 \%$ error show that the beam expansion is more sensitive to positive error in radius than a negative error. This is due to the higher slope of the beam expansion curve toward the shorter wavelength. In addition, an increase in the radius increases the mean dielectric constant by a larger factor than a decrease in the radius decreases its value. With a $-5 \%$ error, the beamwidth increases by less than $30 \%$. Even with 5\% error in the pillar sizes, the overall shape of the curve is intact so a tunable laser can adequately compensate for the error. Such robustness is rarely seen in resonant optical 


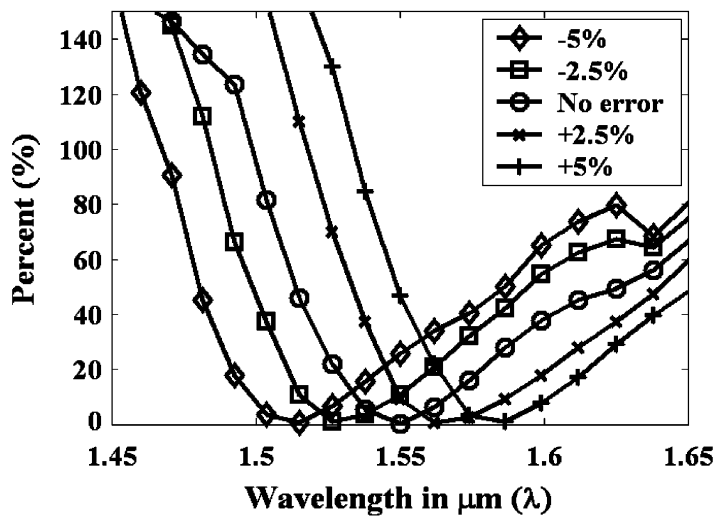

Fig. 6. Beam expansion for systematic error ranging from $-5 \%$ to $5 \%$ showing a shift in the wavelength of minimum beam expansion.

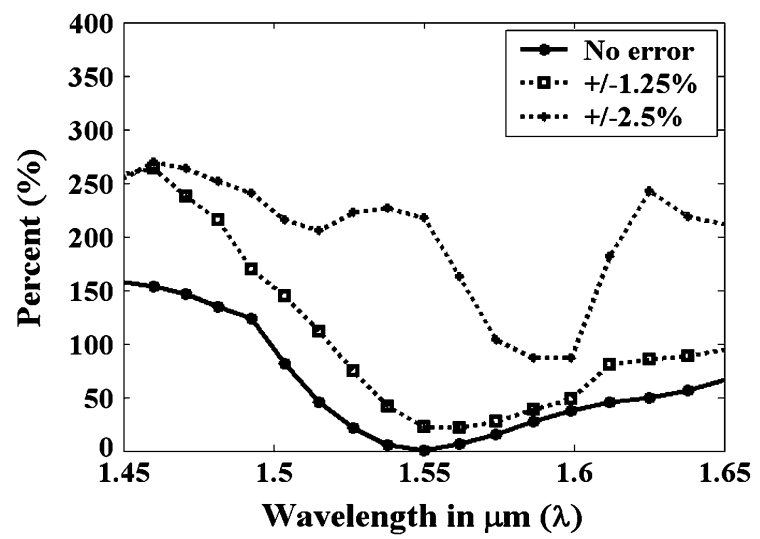

Fig. 7. Beam expansion for pillar radii with a random distribution. Self-collimation remains intact with the $\pm 1.25 \%$ error.

devices where a large Q factor is necessary to achieve good device performance resulting in a very narrowband of operational frequencies [12].

In addition to systematic errors, random errors are simulated. Random errors can occur at any step of the fabrication process, where deviations or fluctuations within a process step can introduce error among the features. Random and normal distributions of the diameter about the mean value at $\pm 2.5 \%$ and $\pm 1.25 \%$ are simulated. For the random distribution, the pillar sizes are uniformly distributed among any value between the two extremes of the range. In the normal distribution, the pillar sizes follow a normal "bell curve" distribution with a standard deviation of $2.5 \%$ and $1.25 \%$, respectively. For each distribution, a set of five simulations are conducted and averaged. The results for the random distribution of pillar sizes are graphed in Fig. 7. The $\pm 1.25 \%$ case retains good collimation with a slight shift toward longer wavelengths, but the $\pm 2.5 \%$ case results in large scattering resulting in the beam not being effectively confined. The shift of the minimum beam expansion wavelength is attributed to the higher average index resulting from the area of the dielectric showing an asymmetric relation to the radius. The results for the normal distribution of error show that with only $1.25 \%$ error, the beam loses its self-collimating characteristic. The normal distribution has a finite probability of pillars that are significantly larger or smaller than the average size. When a

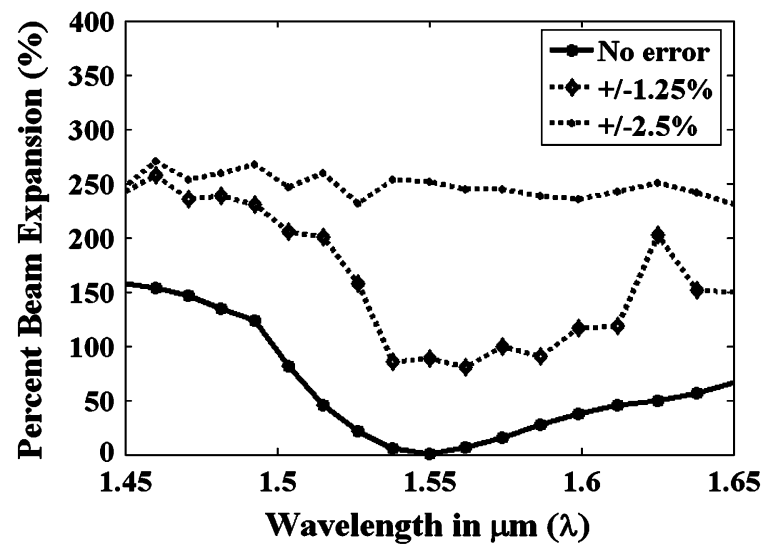

Fig. 8. Beam expansion for pillar radii with a normal distribution. A standard deviation as small as $1.25 \%$ destroys the self collimation.

pillar has high deviation from its surroundings, it acts as an effective scattering center rather than a slight perturbation. Thus, the beam quickly loses its shape with increasing error, as shown in Fig. 8. The analysis of error tolerance reveals that systematic error results in a shifting of the self-collimation wavelength and that small random errors among the pillar sizes are acceptable, but even a small number of pillars with large deviations from the mean size can destroy the performance. $\mathrm{A} \pm 2 \mathrm{~nm}$ range in the pillar diameters can be accommodated, while retaining good self-collimation.

\section{B. Coupling Efficiency Analysis}

Alignment of the optical signal from a fiber or an optical backplane onto an optical chip is difficult and costly requiring specialized connectors and devices to achieve good coupling. Direct fiber to semiconductor waveguide connection is further plagued by the large difference in the mode field size inside of each medium, as well as Fresnel losses from a twofold difference in the index. Photonic crystal virtual waveguides hold a distinct advantage over conventional dielectric waveguides since the propagation inside is analogous to a free-space medium with no dispersion along the specified directions. As long as the beamwidth is significantly larger than the periodicity of the photonic crystal, misalignment will cause no difference in the coupling efficiency.

The tolerance to misalignment is verified by FDTD simulations of a $1.55-\mu \mathrm{m}$ wavelength Gaussian beam propagating from air into a PML terminated photonic crystal by comparing the time-average power through the interface plane to that of the incident beam. Changing the center of incidence along the unit cell results in a deviation of power of less than $0.001 \%$ confirming that a beamwidth of 20 times $a$, the lattice parameter, is sufficiently wide for local alignment with the pillars to have a negligible effect on the beam. A coupled power of $75.6 \%$ was obtained for the coupling coefficient.

To further increase the coupling efficiency, the dielectric constant of the surrounding media is changed and the effect of finite length is investigated. The results in Fig. 9 show that with a higher dielectric constant, the reflected power is decreased due to better index matching. In addition, the reflection coefficient 


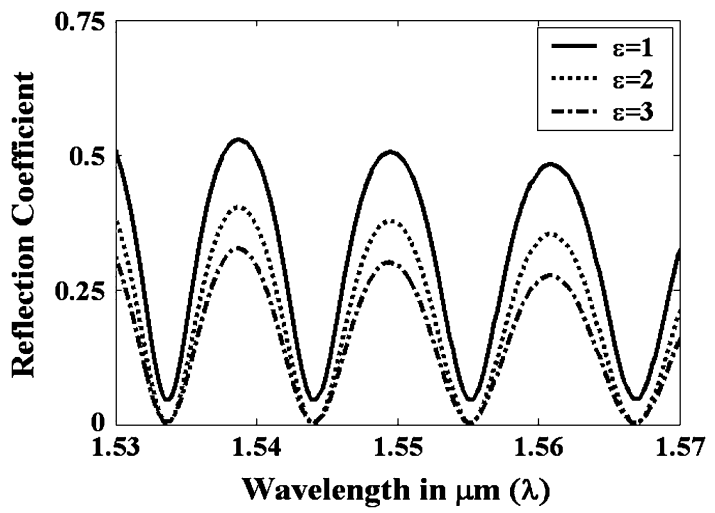

Fig. 9. Reflection coefficient with respect to the outside dielectric constant. The reflection coefficient oscillates to nearly 0 for outside dielectric constant of 2 and 3 for a photonic crystal of length $28.5 \mu \mathrm{m}$.

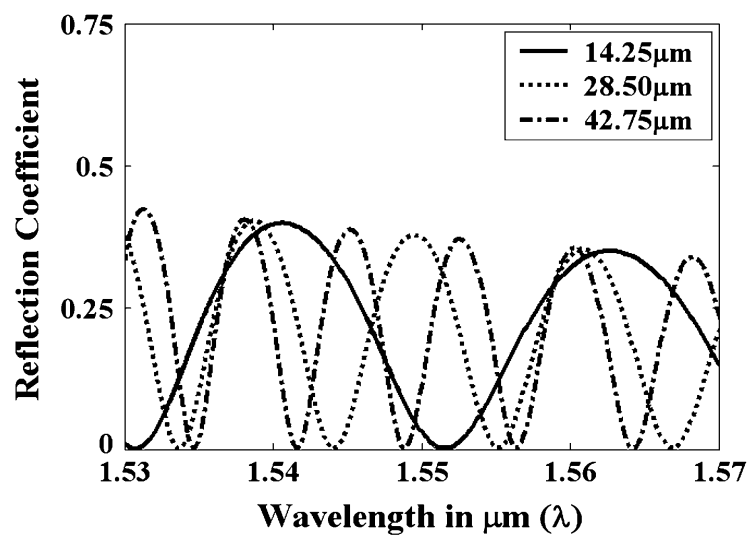

Fig. 10. Reflection coefficient with respect to the length of the photonic crystal for an outside dielectric constant of 2. A longer photonic crystal has a smaller oscillation period.

oscillates from a value close to zero to a maximum reflection value dependent on the wavelength. This phenomenon is analogous to the behavior of a low reflectivity cavity resonator which has a transmission peak spacing in frequency of $\Delta \nu=c / 2 \mathrm{nd}$, where $d$ is the length of the cavity. Therefore, slightly changing the length or the refractive index of the virtual waveguide gives the ability to tune the minimum reflectivity to the design wavelength. An analysis of the reflected power while changing the length of the photonic crystal region, shown in Fig. 10, confirms that the period of the oscillation follows the trend from the resonator analogy.

\section{Evaluation of Routing Functionality}

Optical signals are more difficult to route compared with electrical signals due to restrictions on the radius of curvature of turns in a waveguide. Optical integrated devices are often much larger than their electrical counterparts due to this inability to route signals in a compact manner. Semiconductor waveguides using high-index materials offer better performance, but suffer from difficulties in coupling, such as misalignment and coupling loss from silica and polymer low-index waveguides. Photonic crystal virtual waveguides provide a novel media, ideal for routing optical signals compatible with free-space and low-index waveguides.

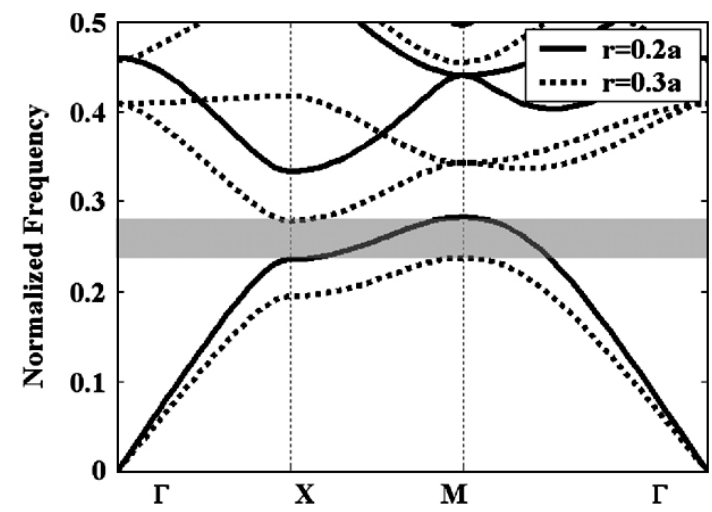

Fig. 11. Band diagram showing the overlap of the bandgap frequencies for the case of $r=0.3$ to the self collimating frequencies for $r=0.2 a$.

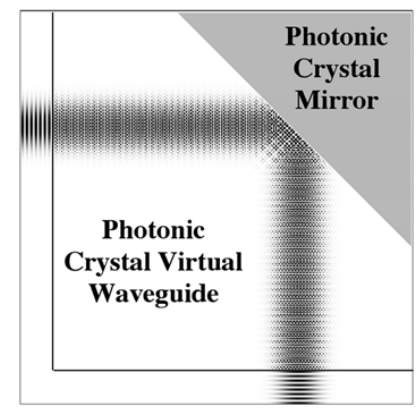

Fig. 12. Sharp $90^{\circ}$ bend in a photonic crystal virtual waveguide through the use of a photonic crystal mirror utilizing the bandgap effect.

One advantage of photonic crystals originates from its symmetric, periodic structure. Rotations of $90^{\circ}$ in square symmetry are invariant, resulting in self-collimated beams traveling in orthogonal directions. This inherent orthogonality creates a convenient Cartesian coordinate system to design the virtual waveguide structure. In addition, the inherent grid system insures that devices and features are perfectly aligned with the propagation direction.

Sharp $90^{\circ}$ turns with no loss are one of the main advantages touted for the photonic crystal line defect waveguide [13]-[15]. Self-collimated beams can also be bent toward a symmetrically equivalent direction through total internal reflection by an interface with a lower index media [7], [8]. A perfect mirror is also formed by a photonic crystal with a bandgap in the frequency range of interest. For the case of a silicon-silica structure, an increase in the radius of the silicon pillars to $0.3 a$ is sufficient to shift the bandgap down to coincide with the self-collimating frequencies of the original structure, as shown in Fig. 11. The bandgap extends between normalized frequencies of 0.24 and 0.28 corresponding to the region of self-collimation in the original structure. By increasing the radius of a diagonal or triangular area intersecting the path of the self-collimated beam, perfect reflection into a perpendicular direction can be accomplished. Fig. 12 shows the structure and the intensity profile of a $1.55 \mu \mathrm{m}$ beam making a $90^{\circ}$ bend. The intensity difference between the input and output beams is due to interference between the incident and reflected beams. The transmission coefficient and the beamwidth expansion after traveling $85.6 \mu \mathrm{m}$ and one 


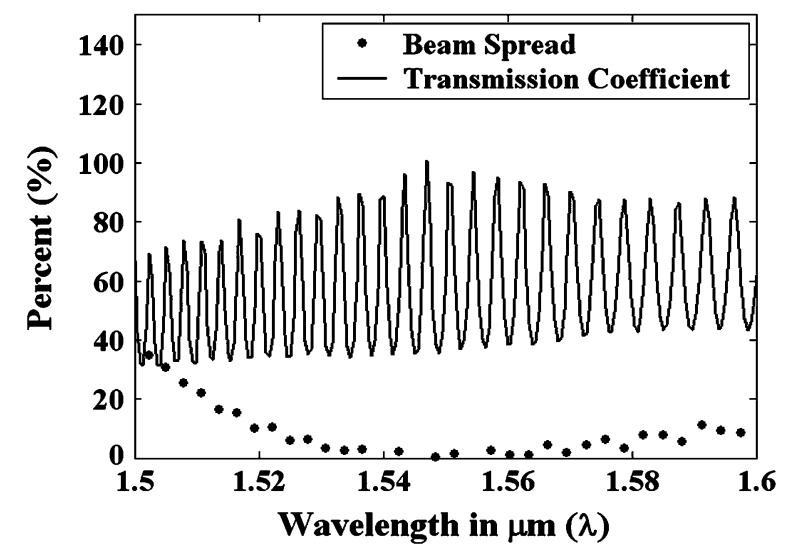

Fig. 13. Beam spread and transmission coefficient for a Gaussian beam traveling through $85.6 \mu \mathrm{m}$ and one $90^{\circ}$ turn in a photonic crystal virtual waveguide.

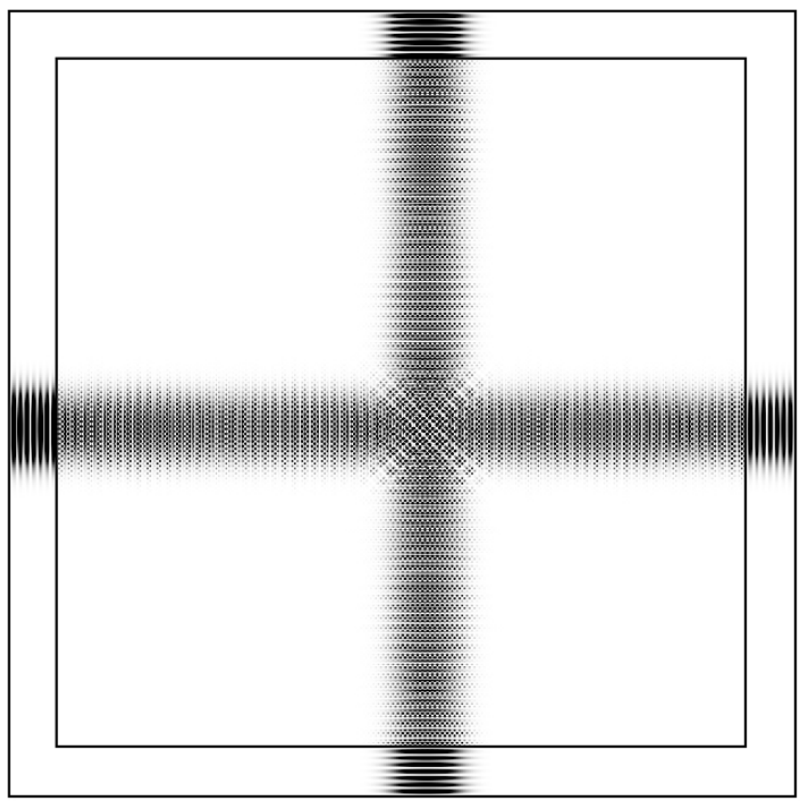

Fig. 14. Noninteracting crossings of self-collimated beams in a photonic crystal virtual waveguide.

$90^{\circ}$ turn are shown in Fig. 13. The beam maintains its collimation and intensity even after reflection from the photonic crystal mirror and shows an oscillation in the transmission coefficient analogous to the straight virtual waveguide.

In addition to sharp turns, the virtual waveguide system has the unique advantage among waveguiding structures of allowing beams to cross each other with no coupling, akin to free-space optics. Fig. 14 illustrates two self-collimated beams traveling through a square photonic crystal virtual waveguide. No coupling occurs between the two beams. This unique combination of guiding and propagation behavior allows compact routing of signals on one optical signal plane rather than having to use complex 3-D structures to avoid signal crossings.

To demonstrate the potential of the virtual waveguide system for routing optical signals, a permutation of three parallel beams switching the output location of the first and third beam is conducted using the FDTD method with the results shown

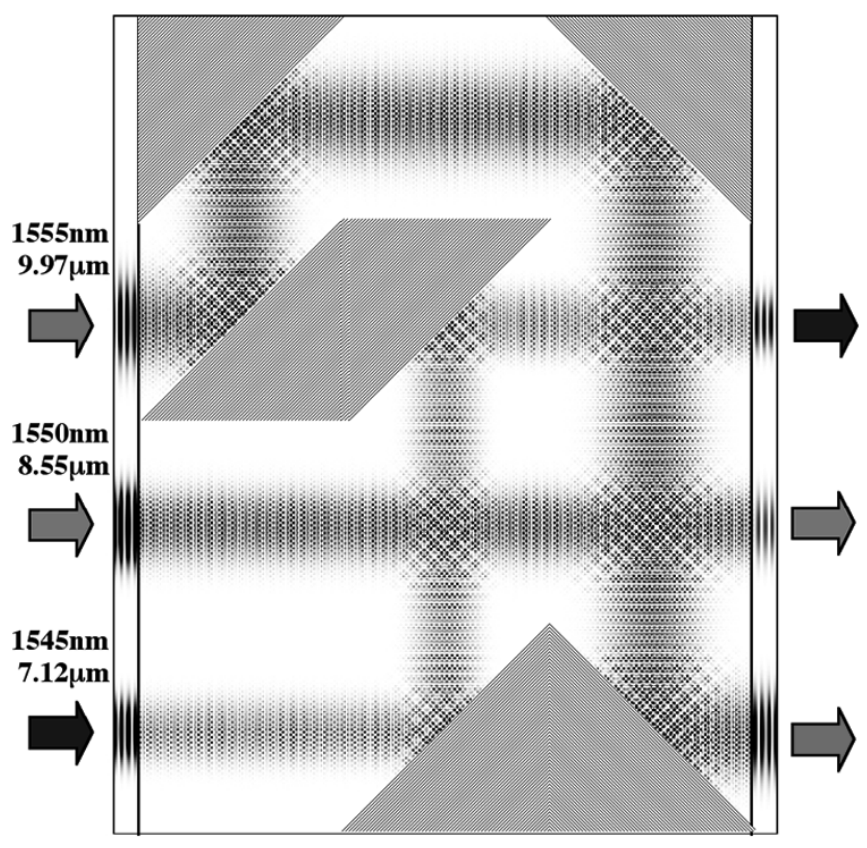

Fig. 15. Routing three parallel beams with different wavelengths and widths within a single signal layer using the photonic crystal virtual waveguide.

in Fig. 15. Beamwidths of $9.97 \mu \mathrm{m}, 8.55 \mu \mathrm{m}$, and $7.12 \mu \mathrm{m}$ along with wavelengths of $1555 \mathrm{~nm}, 1550 \mathrm{~nm}$, and $1545 \mathrm{~nm}$, respectively, are used. This device requires an area spanning only $91.2 \mu \mathrm{m} \times 68.4 \mu \mathrm{m}$ and a single layer as opposed to other waveguiding methods which could take $\mathrm{mm}^{2}$ or even $\mathrm{cm}^{2}$ worth of area and two layers to accomplish the same task.

\section{FUTURE APPLICATIONS}

Photonic crystal virtual waveguides have unique properties with great promise for optical interconnect applications, as well as all-optical devices. Tolerance to misalignment brings the accuracy necessary to achieve good coupling within the limits of the current chip placement and wire bonding technologies. Therefore, methods similar to wire bonding can be applied for low-cost and fast throughput of optical interconnects. Fig. 16 shows a schematic of a configuration where this type of connection can be used. Optical fibers can be directly bonded to the side of a silicon chip where a photonic crystal virtual waveguide carries the signal to the photodetector. Such configurations can dramatically save time and cost compared with conventional methods.

Even though the structure investigated in this paper is a 2-D slab structure, 3-D photonic crystals also exhibit self-collimation and can be used for virtual waveguides. A virtual waveguide utilizing a 3-D photonic crystal with a cubic unit cell can freely route light in the $\mathrm{X}, \mathrm{Y}$, and $\mathrm{Z}$ directions. The capability to route light freely in three dimensions coupled with the ability to cross signals will allow interconnects of the most complex systems.

Finally, by utilizing a material system consisting of nonlinear materials, the mirrors, as well as other features, can be reconfigured and reshaped with an applied electric field. Optical switching and complex optical circuitry can be created, opening the door for a wide range of compact optical devices. 


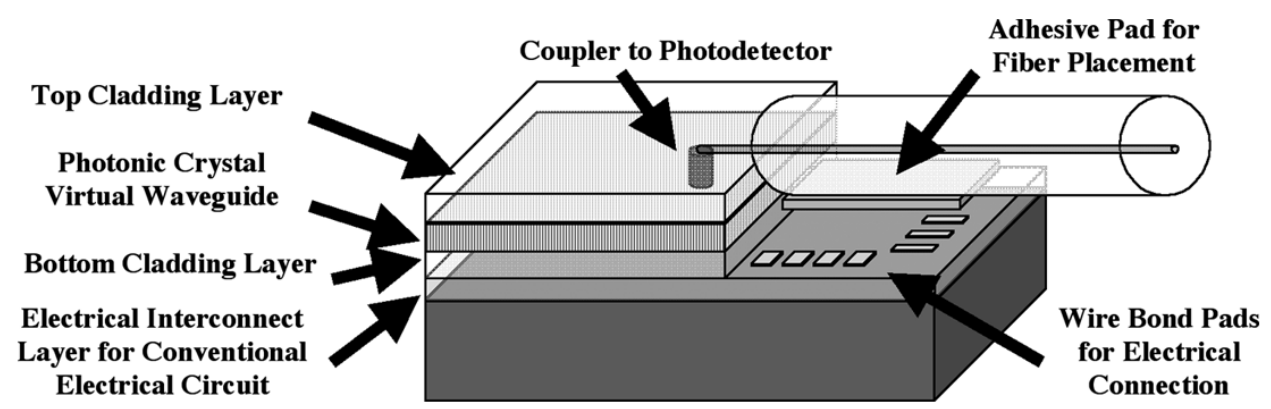

Fig. 16. Low-cost optical interconnect from fiber to a semiconductor chip utilizing the misalignment tolerance of the photonic crystal waveguide.

\section{CONCLUSION}

Photonic crystal virtual waveguides are a novel optical interconnect technology allowing cost effective interconnect from a fiber or the optical backplane to an integrated optical/electrical chip due to its high misalignment tolerance. The self-collimation phenomenon is less sensitive to fabrication error compared with other resonant subwavelength optical devices since it does not rely on a sharp resonance peak to achieve its performance and can be fabricated using the current state of the art technologies. In addition, the ability to perform abrupt $90^{\circ}$ turns and to cross signals with no coupling gives flexibility in the routing circuit layout that is even superior to electrical interconnects. By taking advantage of the resonator-like transmission properties, the coupling coefficient can be improved beyond the limits of Fresnel reflections. Virtual waveguide structures using 2-D and 3-D photonic crystals hold great potential for revolutionalizing integrated optical interconnects, combining the advantages of free-space optics and guided wave optics for complete control of the optical signal.

\section{REFERENCES}

[1] J. D. Joannopoulos, R. Meade, and J. Winn, Photonic Crystals: Molding the Flow of Light. Princeton, NJ: Princeton Univ. Press, 1995.

[2] E. Yablonovitch, "Inhibited spontaneous emission in solid-state physics and electronics," Physical Rev. Lett., vol. 58, pp. 2059-2062, 1987.

[3] S. John, "Strong localization of photons in certain disordered dielectric superlattices," Phys. Rev. Lett., vol. 58, pp. 2486-2489, 1987.

[4] H. Kosaka et al., "Self-collimating phenomena in photonic crystals," Appl. Phys. Lett., vol. 74, pp. 1212-1214, 1999.

[5] J. Witzens, M. Lončar, and A. Scherer, "Self-collimation in planar photonic crystals," J. Sel. Topics Quantum Electron., vol. 8, no. 6, pp. 1246-1257, Nov.-Dec. 2002.

[6] L. Wu, M. Mazilu, and T. Krauss, "Beam steering in planar-photonic crystals: From superprism to supercollimator," J. Lightw. Technol., vol. 21, no. 2, pp. 561-566, Feb. 2003.

[7] X. Yu and S. Fan, "Bends and splitters for self-collimated beams in photonic crystals," Appl. Phys. Lett., vol. 83, pp. 3251-3253, 2003.

[8] C. Chen et al., "Optimized bending efficiency of self-collimated beams in nonchannel planar photonic crystal waveguides," Opt. Express, vol. 11, pp. 3153-3159, 2003.
[9] M. Qui, "Effective index method for heterostructure-slab-waveguidebased two-dimensional photonic crystals," Appl. Phys. Lett., vol. 81, pp. 1163-1165, 2002.

[10] O. Painter, J. Vučković, and A. Scherer, "Defect modes of a two-dimensional phtonic crystal in an optically thin dielectric slab," J. Opt. Soc. Amer. B., vol. 16, pp. 275-285, 1999.

[11] L. Wu et al., "Superprism phenomena in planar photonic crystals," $J$. Quantum Electron., vol. 38, pp. 915-918, 2002.

[12] S. Johnson et al., "Elimination of cross talk in waveguide intersections," Opt. Lett., vol. 23, pp. 1855-1857, 1998.

[13] R. D. Meade et al., "Novel application of photonic bandgap materials: Low-loss bends and high Q cavities," J. Appl. Phys., vol. 75, pp. 4753-4755, 1994.

[14] M. Notomi et al., "Si-based photonic crystals and photonic-bandgap waveguides," IEICE Trans. Electron, vol. E85-C, p. 1025, 2002.

[15] A. Mekis et al., "High transmission through sharp bends in photonic crystal waveguides," Phys. Rev. Lett., vol. 77, pp. 3787-3790, 1996.

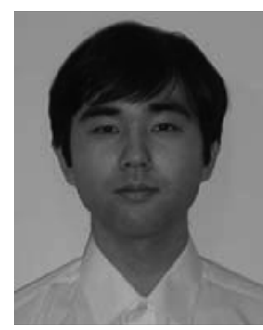

Tsuyoshi Yamashita (S'03) received the B.S. degree in materials science and engineering and the M.S. degree in electrical and computer engineering from the Georgia Institute of Technology, Atlanta, in 2000 and 2004 , respectively. He is currently working towards the Ph.D. degree in the materials science and engineering at the Georgia Institute of Technology researching the simulation and fabrication of photonic crystal devices based on propagation through a photonic crystal.

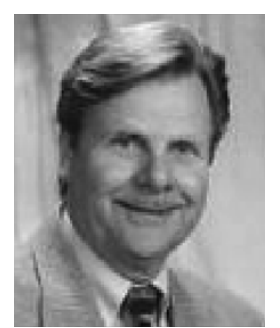

Christopher J. Summers (M'82-SM'94) received the B.Sc. and Ph.D. degrees in physics from Reading University, Reading, U.K.

$\mathrm{He}$ is a Professor in the School of Materials Science and Engineering, Georgia Institute of Technology, Atlanta, and the Director of the Phosphor Technology Center of Excellence. His current research interests in photonic crystals are in propagation through 2-D photonic crystals, such as self collimation phenomena and superprism effects, and the use of 3-D photonic crystals infiltrated using the atomic layer deposition method for luminescence modification. 